\title{
Review Article \\ Optical Spectroscopy for Noninvasive Monitoring of Stem Cell Differentiation
}

\author{
Andrew Downes, Rabah Mouras, and Alistair Elfick \\ School of Engineering, The University of Edinburgh, Edinburgh EH9 3JL, UK \\ Correspondence should be addressed to Andrew Downes, andy.downes@ed.ac.uk \\ Received 25 August 2009; Revised 13 October 2009; Accepted 11 November 2009 \\ Academic Editor: Pierre Bagnaninchi
}

Copyright () 2010 Andrew Downes et al. This is an open access article distributed under the Creative Commons Attribution License, which permits unrestricted use, distribution, and reproduction in any medium, provided the original work is properly cited.

There is a requirement for a noninvasive technique to monitor stem cell differentiation. Several candidates based on optical spectroscopy are discussed in this review: Fourier transform infrared (FTIR) spectroscopy, Raman spectroscopy, and coherent anti-Stokes Raman scattering (CARS) microscopy. These techniques are briefly described, and the ability of each to distinguish undifferentiated from differentiated cells is discussed. FTIR spectroscopy has demonstrated its ability to distinguish between stem cells and their derivatives. Raman spectroscopy shows a clear reduction in DNA and RNA concentrations during embryonic stem cell differentiation (agreeing with the well-known reduction in the nucleus to cytoplasm ratio) and also shows clear increases in mineral content during differentiation of mesenchymal stem cells. CARS microscopy can map these DNA, RNA, and mineral concentrations at high speed, and Mutliplex CARS spectroscopy/microscopy is highlighted as the technique with most promise for future applications.

\section{Introduction}

1.1. Challenges in Stem Cell Science. In current stem cell biology and regenerative medicine, two of the greatest challenges $[1,2]$ are to control the differentiation of stem cells and to ensure the purity of isolated cells. These can both be addressed by careful monitoring and characterization of cells. The process of stem cell differentiation is at present monitored by biological assays, namely, immunocytochemistry $[3,4]$. However, this process is time consuming as well as requiring biomarkers or labels. There is a clear need for a truly noninvasive technique which can monitor the degree of differentiation rapidly. Such a technique will most likely involve a form of optical imaging or spectroscopy but must not involve the addition of any kind of biomarker. Biomarkers are used to sort embryonic stem cells, in conjunction with fluorescent [5, 6] or magnetic [7] labels. These techniques are lengthy and time-consuming, but careful monitoring of stem cell differentiation is essential: in clinical applications, a population of fully differentiated cells is often implanted, but teratomas can result if any stem cells remain undifferentiated [8].
There are a number of issue with the use of biomarkers for the characterization and sorting of stem cells and their derivatives. Firstly, only a limited number of biomarkers exists each one being cell-specific. Many cell types lack biomarkers, for example, cardiomyocytes [9], gastrointestinal stem cells [10], and corneal stem cells [11]. Secondly, the use of biomarkers raises issues with both biological researchers and clinicians, who would strongly prefer a labelfree technique. Finally, these biomarkers cannot easily be translated; for example, embryonic stem cell biomarkers are not always applicable to adult stem cells.

There are further issues with the use of fluorescent and magnetic markers. Fluorescent biomarkers $[5,6]$ have been employed in cell sorting and characterization, but fluorescent techniques have a number of drawbacks. Firstly, photobleaching means that signal levels drop over time; so long-term studies of differentiation are prohibited. Secondly, this process of photobleaching produces free radical singlet oxygen species which will damage live cells. Finally, the use of biomarkers causes modification to cells' surface chemistry, and stem cells are highly sensitive to small changes in their surface chemistry. Magnetic beads cannot easily be visualised 
in microscopy; they must all be removed from the cells; a large mass could cause large mechanical stresses to the cells, which can affect the cells' behaviour.

There is thus a requirement from the stem cell community for a rapid, easy, sensitive, nondestructive, noninvasive, label-free technique which can be applied both on the single cell level and to monitoring or sorting large populations of cells. This review will concentrate on label-free optical spectroscopy techniques, which are noninvasive and have sufficiently high resolution to be applied at the single cell level.

White light imaging-either phase contrast or differential interference contrast (DIC) — can reveal the approximate level of differentiation in situ, to those who are expert in stem cell culture. However, it is only really suitable for monolayers of cells. As white light imaging is usually only qualitative, it would benefit by being replaced by a more advanced optical technique capable of a quantitative measurement on individual cells. Such a technique should therefore be capable of high speed characterization, to enable large numbers of cells to be studied-in monolayer cultures, embryos, and scaffolds.

1.2. Infrared Absorption Spectroscopy. The first optical technique suitable for noninvasive characterization of cells is infrared absorption spectroscopy. Infrared light is absorbed by the wide variety of chemical bonds within molecules, which all have well-defined vibrational frequencies. Hence, an absorption spectrum of a cell should give a characteristic snapshot of the chemistry, and an undifferentiated cell's spectrum could differ enough from that of a differentiated cell enough to characterize them. Simple infrared spectrometers use a broadband light source containing a wide range of wavelengths, which is typically passed through a cuvette of solution, through a dispersing spectrometer onto a single detector. This technique is slow, as the spectrum is built up from around 1000 sequential data points. In order to collect a full spectrum without losing the vast majority of the signal, Fourier transform infrared (FTIR) spectroscopy [12] uses both interferometry and a Fourier transform of the signal: from the time to frequency domain. A typical FTIR setup is illustrated in Figure 1(a), which requires a mirror to scan one half of the interferometer arm over a distance of a few millimetres. A full spectrum is typically acquired in around a second on live cells [13]. Synchrotron sources have promised vastly improved spectral acquisition times — up to 1000 times faster [14] than benchtop FTIR-but it is not clear whether this is applicable to live cells, as heating by absorption may prevent any increase in speed.

The lateral resolution of optical techniques is normally approximated by $0.6 \lambda / \mathrm{N}$.A. where $\lambda$ is the wavelength of illuminating light, and N.A. is the numerical aperture of illumination. Although an N.A. of 1.4 is achievable with objective lenses using visible light, infrared light has very low transmission through standard glass objectives; so a parabolic mirror (known as a Cassegrain objective) is normally used to focus light. These objectives have a typical N.A. of 0.4 . The bonds in molecules are typically

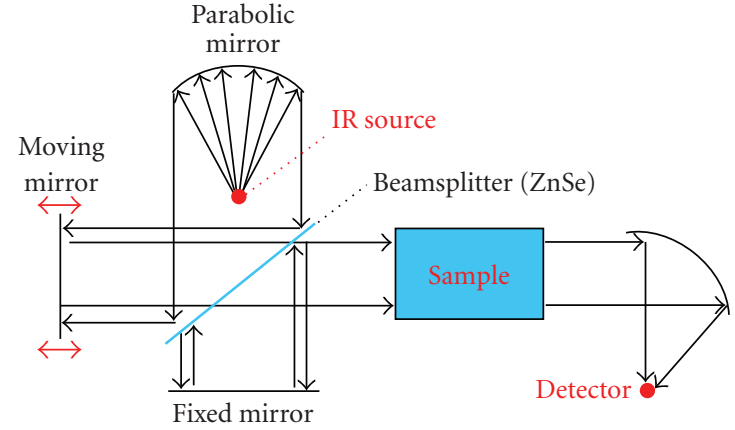

(a) FTIR

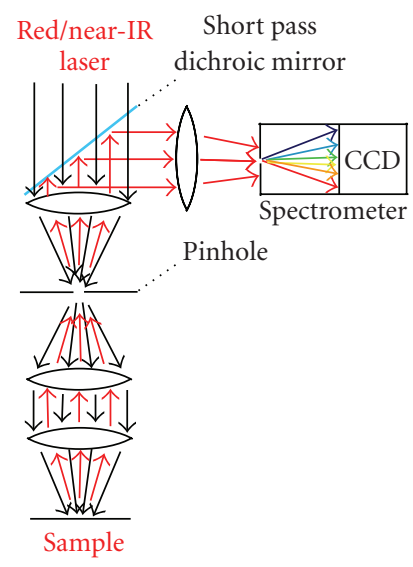

(b) Raman

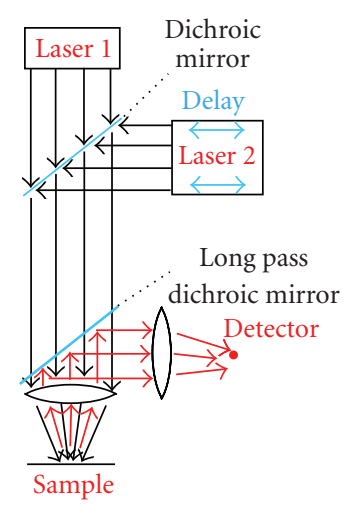

(c) CARS
FIGURE 1: Schematic experimental arrangements for (a) Fourier transform infrared (FTIR) spectroscopy, (b) Raman spectroscopy, and (c) coherent anti-Stokes Raman scattering (CARS) microscopy and spectroscopy.

excited with infrared light of wavelengths between 2.8 and $16 \mu \mathrm{m}$, which corresponds to a lateral resolution of 4.2 to $24 \mu \mathrm{m}$, which is small enough to be applied to individual isolated cells or to average over groups of cells, but will not usually be cell specific when applied to an embryo or group of cells tightly bound together. FTIR microscopy has been achieved on fixed adherent mesenchymal stem cells (MSCs) [15] with a diameter of around $50 \mu \mathrm{m}$, but only for high-frequency (short wavelength) vibrations. These vibrational frequencies are described by spectroscopists as inverse wavelengths in units of $\mathrm{cm}^{-1}$ ["wavenumbers"]. The lowest frequency vibrations occur in cells around $600 \mathrm{~cm}^{-1}$ $(\lambda=16.7 \mu \mathrm{m})$ and the highest frequencies relate to the $\mathrm{C}-\mathrm{H}$ stretch $\left(2800-3000 \mathrm{~cm}^{-1}, \lambda=3.3-3.6 \mu \mathrm{m}\right)$ and $\mathrm{O}-\mathrm{H}$ stretch $\left(\sim 3500 \mathrm{~cm}^{-1}, \lambda=2.8 \mu \mathrm{m}\right)$.

One of the major issues with infrared radiation is its extremely low penetration depth in water, which limits the depth which can be probed in solution to the range 10$100 \mu \mathrm{m}$. To combat absorption of infrared light through a thick cuvette, attenuated total reflection- (ATR-) FTIR [16] probes only the first micrometre above the substrate. An array of spectra can be acquired rapidly enough to perform imaging within a few minutes on live cells [17]. Results from FTIR on stem cells will be discussed later. 


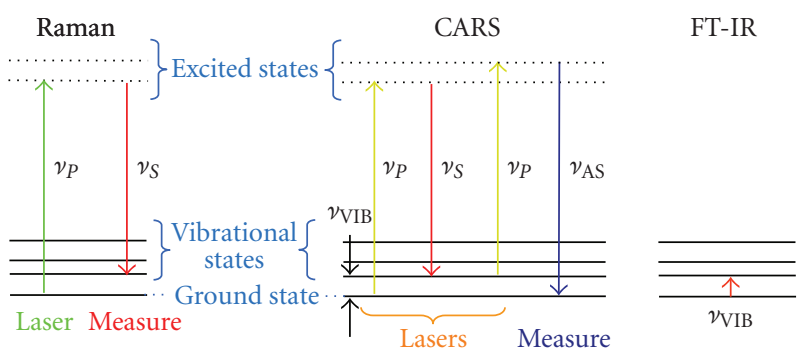

FIgURE 2: Schematic energy level diagrams for Raman, CARS, and FTIR processes. In Raman scattering one illuminating laser photon is absorbed (at pump frequency $\nu_{P}$ ), and another is radiated (at Stokes frequency $v_{S}$ ) - the difference in frequencies being equal to a vibrational frequency, $\nu_{\mathrm{VIB}}$. In CARS three photons are absorbedtwo at frequency $\nu_{P}$ and one at $\nu_{S}$-and one is emitted at the anti-Stokes frequency $\nu_{\mathrm{AS}}$. FTIR relies solely on the absorption of infrared radiation at $\nu_{\mathrm{VIB}}$.

1.3. Raman Spectroscopy. The second optical technique suitable for stem cell characterization is Raman spectroscopy [18]. In the most widespread form-Stokes scatteringvisible or near-infrared light loses energy (frequency) by exciting molecules into their excited state, as depicted in Figure 2. This means that some of the laser light is red-shifted after interacting with the sample. A typical setup is illustrated in Figure 1(b): after filtering out the laser, the remaining red-shifted light is passed through a spectrometer onto a cooled CCD camera. A full Raman spectrum is normally acquired in 1 to 10 seconds, so is typically slower than FTIR. As silicon CCDs have a response which dies away rapidly at a wavelength of around $1000 \mathrm{~nm}$, the longest laser wavelength which can be used is $785 \mathrm{~nm}$ (AlGaAs diode) which is the preferred wavelength for Raman spectroscopy in biology. The strong $\mathrm{C}-\mathrm{H}$ stretch frequencies are the highest measurable with such a laser wavelength. The other popular laser wavelength for biological samples is $633 \mathrm{~nm}$ (HeNe laser) which has lower power than $785 \mathrm{~nm}$ lasers, and heating in cells and tissue is the lowest using nearIR illumination (700-1100 nm). Heating has been measured directly in cells-using $100 \mathrm{~mW}$ of $1064 \mathrm{~nm}$ radiation in an optical trap, a temperature rise of $<1^{\circ} \mathrm{C}$ was observed [19]. Prolonged exposure to $300 \mathrm{~mW}$ illumination at constant power ("continuous wave", CW) caused photodamage-a light-induced reduction in cell viability-attributed to twophoton absorption. Visible light $(\lambda<700 \mathrm{~nm})$ is believed to cause photodamage at far lower thresholds than this, due to increased absorption by proteins, but shorter wavelengths produce more Raman signal [20].

The reduction in wavelengths for Raman spectroscopy, compared to FTIR, equates to a greatly improved lateral resolution of $350 \mathrm{~nm}$ and axial (depth) resolution of $1150 \mathrm{~nm}$. This is sufficient to allow spectroscopy on individual cells, and even at the subcellular level. Furthermore, Raman microspectroscopy-otherwise known as Raman mapping or Raman microscopy—can be performed [21]. Maps can be produced containing the total signal under a given peak, or more subtle differences between spectra can be exploited with principal component analysis (PCA) [22] or cluster analysis. This reduces the large number of peaks in the Raman spectrum to a smaller number of independent variables, and cluster analysis produces colour-coded maps with regions of similar chemistry deduced from the set of spectra. Due to the long acquisition time required, only Raman microscopy of fixed cells had been performed until recently, as live cells move far more quickly than the set of spectra could be recorded. However, recently Raman microscopy has been performed on live cells with $100 \mathrm{~mW}$ power at $647 \mathrm{~nm}$ [23] and acquisition time of 0.5 second, albeit with only $32 \times 32$ pixels. An alternative approach is to illuminate a line rather than a spot-then a series of spectra can be recorded across a single CCD, each relating to a pixel along the line. This technique has been applied to live cells [20] with 5 seconds per line ( 3 minutes per image) with $532 \mathrm{~nm}$ at $3.5 \mathrm{~mW} / \mu \mathrm{m}^{2}$ intensity, again with a small number of imaging pixels. It remains to be seen whether photodamage was occurring in both of these live cell imaging publications.

PCA can be used to extract the most significant variations between groups of spectra acquired on large numbers of cells. Thus, determining an unknown cell type from two possibilities-notably, stem cell and differentiated cell—can be accomplished using large numbers of spectra from known cell types. The most important differences are highlighted, rather than any uncorrelated and unimportant variations, to improve the sensitivity of the technique. No knowledge of the chemistry is required with this unsupervised technique. Two improved variants of PCA are discussed in relation to FTIR, and both show marked improvements in their ability to distinguish cell types [24]. Before analysis can be performed, spectra require processing to remove unwanted autofluorescence from tissue-normally by baseline subtraction, as well as the removal of cosmic rays, and substrate and media contribution to the spectrum. All this processing and analysis of spectra requires significant computing power, when applied to large datasets.

Raman spectra consist of a large number of peaks at well-defined frequencies, as demonstrated in Figures 3 and 4. The relative intensities of the peaks change between cells, but the frequencies themselves do not shift. Peaks are usually around $5-10 \mathrm{~cm}^{-1}$ in width, except for the single peak at $1002 \mathrm{~cm}^{-1}$. This frequency relates to the in-plane vibration of the aromatic ring of the phenylalanine molecule which is highly symmetric and is narrower than the other peaks because of the lack of vibration out of the plane and lack of variation in other atoms attached to the benzene ring. Frequencies in FTIR can be slightly shifted by a few $\mathrm{cm}^{-1}$ - and tend to be broader. Both techniques excite these vibrations to different extents; so the relative peak intensities will be different in Raman and FTIR spectra.

1.4. CARS Microscopy and Spectroscopy. Raman scattering is a weak process-typically only 1 in $\sim 10^{10}$ incident photons gives rise to a Raman-shifted photon. This is largely due to the excitation of the bond vibration far above its resonant frequency. In order to increase this efficiency, in coherent anti-Stokes Raman scattering (CARS) [25-28] the vibration 
is excited with two laser frequencies-the difference (or beat) frequency between them is matched to the vibrational frequency of interest. This gives between 4 and 6 orders of magnitude more signal than standard Raman. This means that CARS images are acquired in seconds, whereas a similar quality Raman map would require days to complete. The process is best excited with pulsed lasers with durations of around $6 \mathrm{ps}$, which should mean that no photodamage occurs with extensive use of powers of at least $12 \mathrm{~mW}$ [29]. A CARS microscope, designed for biological imaging, is described in detail elsewehere [28], which has a lateral resolution of $350 \mathrm{~nm}$ and an axial resolution of $1100 \mathrm{~nm}$. Live cell imaging is slightly slower than that of fixed cells, but high-quality images are acquired within 1 minute.

Picosecond CARS excitation pulses have an estimated width of around $3 \mathrm{~cm}^{-1}$, so are ideal for biological molecules, but this means that only one vibration (spectral peak) may be excited during an image. Images can be acquired sequentially at different wavenumbers, by retuning one laser, but this is not ideal given the motion occurring during live cell imaging. Some CARS systems are able to acquire images at two different vibrational frequencies simultaneously [28]. Multiplex CARS [30-34] uses normal (narrowband) pulses for one laser, $v_{P}$, and broadband pulses for $v_{S}$. The broadband supercontinuum excitation pulse is several hundred nanometres wide and is excited in a photonic crystal fibre by a femtosecond laser. A full spectrum is acquired in around 100 milliseconds on live yeast [30], but an estimate of photodamage [29] suggests that 1 second per pixel would be more appropriate for eukaryotic cells. Further improvements to excitation sources could see this fall to the millisecond range-enabling high-quality, noninvasive full spectral mapping of live cells in minutes.

A different approach to increase the speed of Raman microscopy, termed Stimulated Raman Scattering, has recently been published [35]. In a similar way to how stimulated emission depopulates the excited state in lasers, the excited state in a simple Raman excitation (pumped with $\nu_{P}$ ) can be rapidly depopulated by a second laser (at $\nu_{S}$ ) modulated in the $\mathrm{MHz}$ range. In this way, the signal at $v_{S}$ is increased slightly (Stimulated Raman Gain) and the pump power at $\nu_{P}$ is decreased slightly (Stimulated Raman Loss, SRL). Monitoring either signal, filtered by a lock-in amplifier, produces images which are background-free and directly proportional to the concentration. The standard CARS signal has a quadratic dependence on concentration and can have a large unwanted background. Heterodyne CARS [36] is another technique which is able to circumvent both of these problems encountered in standard CARS imaging. SRL is a linear optical technique and is suitable for extension into optical coherence tomography deep into tissue, using low numerical aperture lenses [37].

\section{Results and Discussion}

2.1. Fourier-Transform Infrared (FTIR) Spectroscopy. FTIR spectroscopy was used to study murine embryonic stem cells by Ami et al. [38]. After 4-7 days of differentiation, changes to the absorption spectrum of fixed cells were noticed: features in the amide I band $\left(1600-1700 \mathrm{~cm}^{-1}\right)$ were enhanced, and those in the nucleic acid region (850$1050 \mathrm{~cm}^{-1}$ ) diminish. This means that the overall levels of DNA and RNA decrease, and the alpha helix content of proteins increases over time. Furthermore, new DNA/RNA hybrid bands at $899 \mathrm{~cm}^{-1}$ and $954 \mathrm{~cm}^{-1}$ start to occur around day $4-7$, suggesting that mRNA translation is occurring at this time.

German et al. [11] employed high-intensity synchrotron radiation to probe $10 \mu \mathrm{m}$ thick cryosections of bovine cornea. They used PCA to clearly distinguish the three cells types of interest: stem cells, transit-amplifying cells, and terminal differentiated cells. No biomarkers of corneal stem cells exist; so spectroscopic techniques offer the only viable method of cell characterization here.

From the same group, Walsh et al. [39] again used synchrotron FTIR, this time on paraffin-embedded human intestinal crypts, which were dewaxed. The position of cells along the crypt denotes the change from stem cell location to transit-amplifying region to differentiated location. PCA was used to compare spectral features and was able to separate cell types from three positions along the crypt, which is shown in Figure 3. This method of characterization was compared with tissue stained with two different immunophenotypical markers: rabbit polyclonal anti-CD133 and $\beta$-catenin antibodies. The authors state that the dominant FTIR absorption peak at $1080 \mathrm{~cm}^{-1}$, relating to the symmetric $\left(\mathrm{PO}_{2}\right)^{-}$stretch, is a more robust marker than the two biomarkers. As gastrointestinal stem cells lack specific biomarkers, they went on to compare FTIR data against a number of chemical differences which are discussed at length in a further publication [10].

Salasznyk et al. [41] used FTIR to study osteoblasts derived from human mesenchymal stem cells after 28 days of cell culture. Samples were dried and ground into a powder, then pressed into a pellet. The spectrally derived mineralto-matrix ratio was calculated as the ratio of the integrated areas of the phosphate absorbance $\left(900-1200 \mathrm{~cm}^{-1}\right)$ and protein amide I band $\left(1585-1720 \mathrm{~cm}^{-1}\right)$. They observed a significant decrease in the mineral-to-matrix ratio in the extracellular matrix produced by focal adhesion kinase(FAK-) knockdown cells when compared to untreated (control) cells. These FTIR results are compared favourably with biochemical assays.

Krafft et al. [15] also used FTIR to study human mesenchymal stem cells differentiating into osteoblasts. Their samples were fixed in methanol then dried, and they were able to distinguish cells stimulated in osteogenic medium for 7 days, from nonstimulated cells. FTIR microscopy on isolated adherent cells (of size $\sim 50 \mu \mathrm{m}$ ) showed that some of the nonstimulated cells had high levels of glycogen accumulation, and some stimulated cells had a high expression of calcium phosphate. Stimulated cells had reduced levels of amide I (at $1631 \mathrm{~cm}^{-1}$ ), meaning that lower concentrations of beta-sheet proteins were present. This compares well with Ami et al. [38], who measured a higher alpha helix proportion during differentiation. Nucleic acids were hard to detect in this study. 


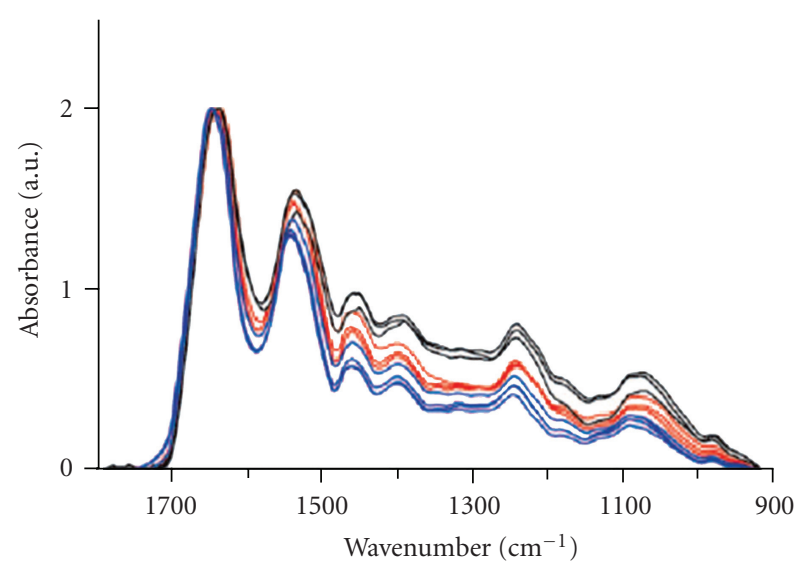

(a)

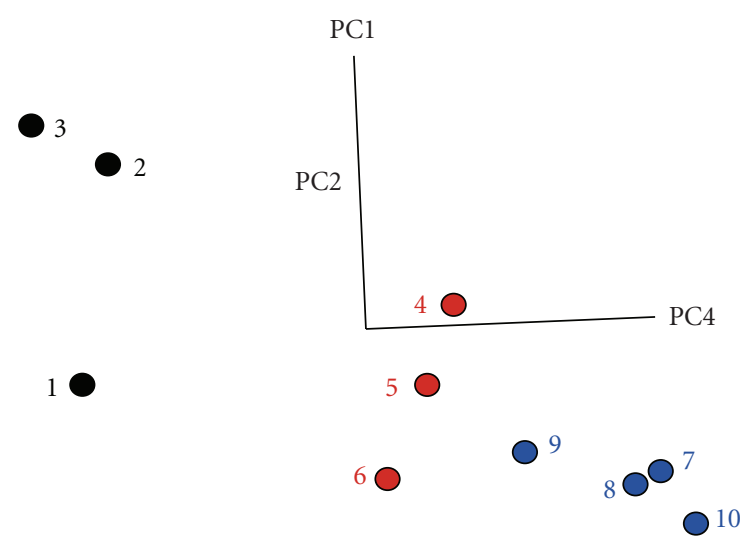

(b)

FIGURE 3: IR spectral analysis of a small intestinal crypt using synchrotron FTIR microspectroscopy. (a) Ten IR spectra of the entire biochemical-cell fingerprint region (900 to $1,800 \mathrm{~cm}^{-1}$ ) acquired from the assigned transit-amplifying location (locations 1-3; black lines, top), the putative stem cell location (locations 46; red lines, middle), and the differentiated location (locations 7 10; blue lines, bottom). (b) PC analysis of a small intestinal crypt's IR spectra using the entire biochemical-cell fingerprint region. Reprinted with permission from [39] (Copyright AlphaMed press 2008).

Bentley et al. [42] measured FTIR spectra of human corneal stem cells and their derivatives: transit amplifying cells. They made $10 \mu \mathrm{m}$ thick cryosections of cornea, which were left to dry and observed differences in synchrotron FTIR spectra which were attributed to nucleic acids. They were able to distinguish between both cell types with spectra acquired from two different regions in the tissue, using PCA, albeit with an overlap of $16 \%$ between the two populations.

In summary, FTIR is able to distinguish between stem cells and their derivatives. Using synchrotron sources, the speed of data acquisition and the quality of comparison have improved greatly. Even microscopy has been performed, requiring a spectral acquisition at each imaging pixel. To be able to distinguish between individual cells, rather than populations, requires subconfluent adherent cells due to the spatial resolution (governed by the wavelength of infrared radiation) being of the order of the cell size. The technique has until now been limited to dried samples due to the high absorption coefficient of water; so the technique has not been used to noninvasively monitor stem cell differentiation in vitro as the required drying is clearly destructive. However, ATR-FTIR can be used on live cell cultures; hence it could potentially be employed as a real-time noninvasive technique for monitoring stem cell differentiation on adherent cells (though no results have yet been published). Such a technique would be highly preferable to the use of biomarkers in clinical as well as research applications. One issue with ATRFTIR is that it only probes the first $1-2 \mu \mathrm{m}$ above a substrate; so the nucleus would only give a small contribution to the overall signal. Given that nucleic acids play a large part in the reported changes in spectral signatures during differentiation, and the nucleus may remain above this penetration depth, it remains to be seen whether ATR-FTIR can indeed be used as a noninvasive biomarker-free analytical technique for live cell studies.

2.2. Raman Spectroscopy. Notingher et al. [43] used Raman spectroscopy to investigate live murine embryonic stem cells. $100 \mathrm{~mW}$ of $785 \mathrm{~nm}$ laser light was focussed onto a spot of size $10 \mu \mathrm{m} \times 5 \mu \mathrm{m} \times 25 \mu \mathrm{m}$. The group grew cells on a gelatinecoated quartz substrate, as plastic contains a number of vibrational bonds which are also present in cells. Glass gives a strong fluorescence background; so quartz or magnesium fluoride is preferred as a substrate. However, stem cells do not adhere well to glass and crystals; so a thin coating to the substrate is required, such as the gelatine used in this study. Great care must also be taken to ensure that the stem cells do not differentiate spontaneously on a given substrate or coating. Over 16 days of differentiation, they observed a decrease in the RNA peak (at $813 \mathrm{~cm}^{-1}, \mathrm{O}-\mathrm{P}-\mathrm{O}$ stretch) by $75 \%$ [40] and a drop of $50 \%$ in the DNA peak (at $788 \mathrm{~cm}^{-1}$, cytosine ring vibration). Peaks were normalized to the total Raman signal. They also extracted the first principal component spectrum, reproduced in Figure 4, which reveals the spectrum responsible for most of the differences between spectra of stem cells and differentiated cells. Note the high coincidence of many peaks of this principal component spectrum, with the spectrum of RNA. This confirms that a reduction in RNA levels dominates the chemical changes during differentiation.

Chan et al. [9] acquired Raman spectra on live human embryonic stem cells (hESCs) as they differentiated into cardiomyocytes and were able to distinguish between hESCs and hESC-derived cardiomyocytes with an accuracy of $66 \%$. They found that the RNA peak $\left(811 \mathrm{~cm}^{-1}\right)$ and DNA peaks (e.g., $785 \mathrm{~cm}^{-1}, 1090 \mathrm{~cm}^{-1}$ ) were all reduced in intensity during differentiation.

We acquired data which is previously unpublishedRaman spectra of fixed mesenchymal stem cells grown on gelatine-coated quartz, and used a diffraction-limited laser spot, instead of averaging over a large area. This meant that spectra could be acquired separately from within the nucleus and within the cytoplasm-these spectra are shown in Figure 5. The spectrum from the substrate has been 


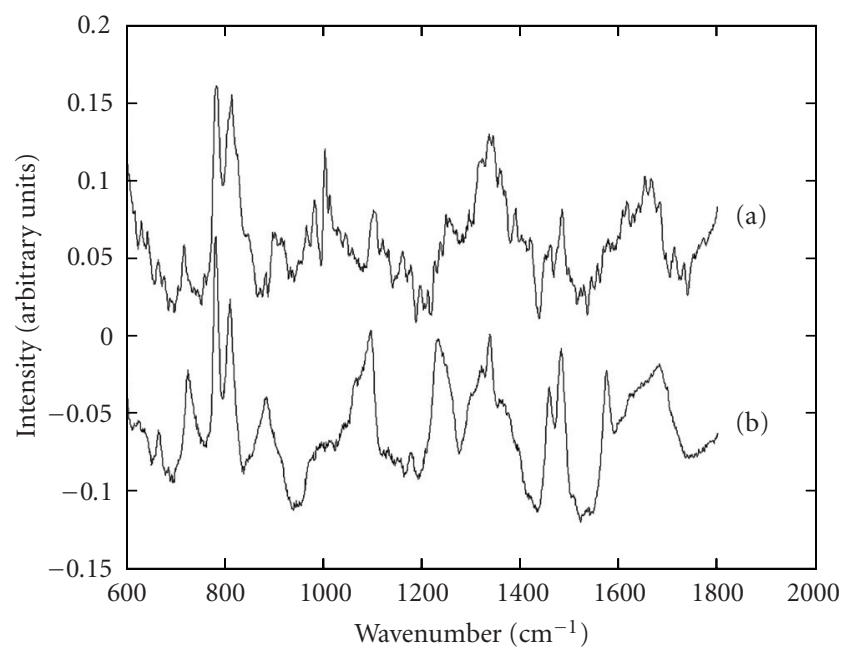

FIGURE 4: (a) First principal component, describing the major differences between two groups: undifferentiated murine embryonic stem cells and the differentiated cells via formation of embryoid bodies. (b) Raman spectrum of reference RNA, revealing a good deal of similarity with (a) - hence, the major change to the spectrum during differentiation is related to a reduction in RNA levels. Note the strong peaks around $785 \mathrm{~cm}^{-1}$ (cytosine and uracil ring stretching), $811 \mathrm{~cm}^{-1}$ (phosphodiester bond stretching), and $1096 \mathrm{~cm}^{-1}$ (phosphodioxy group stretching). All spectra were acquired in 2 minutes (Reprinted with permission from [40]; Copyright 2004 American Chemical Society).

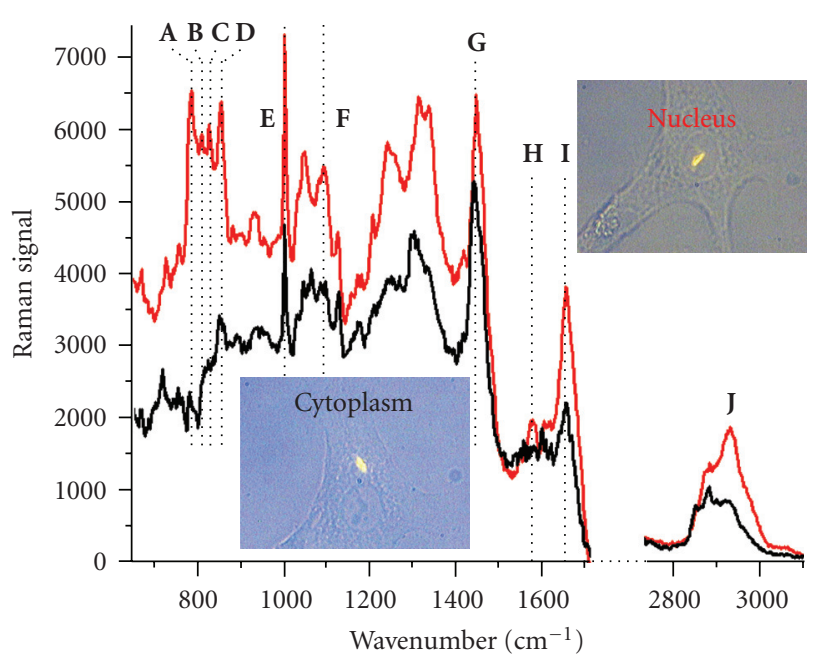

FIGURE 5: Individual Raman spectra within a single mesenchymal stem cell (red: nucleus, black: cytoplasm). The following peaks are specific to DNA and RNA: A $\left(785 \mathrm{~cm}^{-1}\right.$, uracil/cytosine/thymine ring breathing, $\mathrm{O}-\mathrm{P}-\mathrm{O}$ stretch $), \mathbf{B}\left(813 \mathrm{~cm}^{-1}, \mathrm{O}-\mathrm{P}-\mathrm{O}\right.$ stretch $), \mathbf{C}$ $\left(828 \mathrm{~cm}^{-1}, \mathrm{O}-\mathrm{P}-\mathrm{O}\right.$ antisymmetric stretch $), \mathbf{F}\left(1093 \mathrm{~cm}^{-1}, \mathrm{O}-\mathrm{P}-\mathrm{O}\right.$ stretch and $\mathrm{C}-\mathrm{C}$ stretch), and $\mathbf{H}\left(1580 \mathrm{~cm}^{-1}\right.$, adenine/guanine $\mathrm{C}-\mathrm{N}$ stretch). Peaks specific to proteins are D $\left(854 \mathrm{~cm}^{-1}\right.$, tyrosine ring breathing), $\mathrm{E}\left(1004 \mathrm{~cm}^{-1}\right.$, phenylalanine ring breathing), and I $\left(1660 \mathrm{~cm}^{-1}\right.$, amide I alpha helix). Peaks dominated by lipids are G $\left(1448 \mathrm{~cm}^{-1}, \mathrm{CH}_{2}\right.$ deformation) and $\mathbf{J}\left(2800-3000 \mathrm{~cm}^{-1}, \mathrm{C}-\mathrm{H}\right.$ stretch). subtracted, but no baseline subtraction was performedto highlight the requirement for automated subtraction in quantitative spectroscopic analysis. Small variations in laser power and focus position are thought to be responsible for the offset spectra at low wavenumbers. The spectra in Figure 5 as expected clearly demonstrate that the nucleus contains far more DNA and RNA than does the cytoplasm; and that the cytoplasm contains far more proteins and lipids than the nucleus. These cells were fixed rather than live, but a study of fixation methods on Raman spectra [44] indicates that the effect of aldehyde cross-linking fixation on spectra is minimal. This study, together with the data from Notingher et al. [40, 43], implies that it is the nucleus size (or nucleic acid density therein) which shrinks during differentiation. Hence, monitoring the nucleus size in a quantitative manner-using imaging processing techniques - would seem to be a good potential approach to monitoring the state of differentiation.

Mesenchymal stem cells were monitored by Raman spectroscopy during differentiation into osteoblasts [45]. Mineralization was monitored at two frequencies: $960 \mathrm{~cm}^{-1}$ (P-O stretch) and $1070 \mathrm{~cm}^{-1}\left(\mathrm{PO}_{4}{ }^{3-}\right)$. The $960 \mathrm{~cm}^{-1}$ peak relates to the mineral hydroxyapatite- $\mathrm{Ca}_{5}\left(\mathrm{PO}_{4}\right)_{3}(\mathrm{OH})-$ and was by far the strongest signal; this peak height rose linearly from zero to the dominant peak in the spectrum over the 21 days of differentiation. The peak at $1030 \mathrm{~cm}^{-1}$ for $\left(\mathrm{CO}_{3}{ }^{2-}\right)$ remained constant throughout.

Pelled et al. [46] used Raman spectroscopy to compare tissue-engineered bone derived from mesenchymal stem cells, with femoral bone. They found a very good similarity in phosphate $\left(960 \mathrm{~cm}^{-1}\right)$ and carbonate $\left(595 \mathrm{~cm}^{-1}\right)$ levels, with only minor spectral differences such as a larger amount of protein. Liu [47] also observed a major peak at $960 \mathrm{~cm}^{-1}$ due to hydroxyapatite, in mineral extracted from odontoblast nodules - which were formed by the differentiation of dental pulp stem cells.

Azrad et al. [48] used Raman spectroscopy to characterize the production of mineral content from mesenchymal stem cells, under the influence of two osteogenic agents: quality elk velvet antler (QEVA) extract, and dexamethasone. They measured no mineralization from the control group, some mineralization from the dexamethasone-fed cells, but most mineralization from cells supplemented with the elk velvet antler. Peaks indicated phosphate derivatives, which for QEVA were mostly related to hydroxyapatite (at $960 \mathrm{~cm}^{-1}$ ) and its precursors (amorphous calcium phosphate, $\mathrm{Ca}_{9}\left(\mathrm{PO}_{4}\right)_{6} \cdot \mathrm{H}_{2} \mathrm{O}$, at $952 \mathrm{~cm}^{-1}$ and octacalcium phosphate, $\mathrm{Ca}_{8}\left(\mathrm{PO}_{4}\right)_{6} \cdot 5 \mathrm{H}_{2} \mathrm{O}$, at $\left.957 \mathrm{~cm}^{-1}\right)$. For dexamethasonefed cells, the dominant peak was of octacalcium phosphate, and lower amounts of hydroxyapatite and amorphous calcium phosphate were measured.

Gentleman et al. [49] compared mineralized nodules in vitro from 3 sources: embryonic stem cells, neonatal calvarial osteoblasts, and adult bone-marrow-derived mesenchymal stem cells. After 28 days in osteogenic medium, sets of Raman spectra were acquired of the mineralized nodules, and PCA was performed on the spectra to extract their chemical components. The osteoblasts and mesenchymal stem cells both produced nodules which were chemically 
similar to native bone: a combination in descending order of concentration, of (a) carbonate-substituted hydroxyapatite, (b) crystalline nonsubstituted hydroxyapatite, and (c) amorphous phosphate species. However, embryonic stem cells produce nodules which were dominated only by the first mineral component, which was similar to synthetic carbonated hydroxyapatite. Nanoindentation tests showed that these nodules derived from embryonic stem cells were more than an order of magnitude less stiff than those derived from osteoblasts and mesenchymal stem cells.

These Raman studies all show a similar ability to distinguish stem cells from their derivatives, that is, a similar sensitivity, as the FTIR technique. In addition, mineralization can be monitored with great subtlety. The major advantage of FTIR over Raman is its speed; hence mapping signals (derived from a full spectral acquisition at each imaging pixel) into images is easier with FTIR. The major advantage of Raman over FTIR is that has been used on live cells, so is truly noninvasive.

2.3. CARS Microscopy and Spectroscopy. CARS microscopy has been performed on live murine embryonic stem cells by Konorov et al. [50], but the laser setup only permitted pixel dwell times of 300 milliseconds compared to microseconds in standard CARS microscopy [25-28]. Hence the image quality was poor- the groups were unable to distinguish any individual cells or features when imaging at the DNA and RNA frequencies. However, CARS spectroscopy showed a large reduction in the RNA peak intensity $\left(\right.$ at $\left.811 \mathrm{~cm}^{-1}\right)$ in differentiated cells.

Figure 6 shows our CARS microscopy image, acquired on MCF-7 human breast cancer cells which have a similarly large nucleus to cytoplasm ratio as stem cells. Two sequentially acquired images are overlaid: in green, DNA/RNA is mapped at the phosphate backbone $\mathrm{O}-\mathrm{P}-\mathrm{O}$ stretch frequency $\left(1095 \mathrm{~cm}^{-1}\right)$, and in red, lipids and the cytoskeleton are mapped at the $\mathrm{CH}_{2}$ deformation frequency $\left(1448 \mathrm{~cm}^{-1}\right)$. The $\mathrm{O}-\mathrm{P}-\mathrm{O}$ stretch frequency is also present in Figure 4(a)the principal component spectrum which describes most of the changes during differentiation-and in Figure 4(b)the RNA spectrum. This method can be easily extended to monitoring the nucleus size in live stem cells, with a reduced frame rate of at least 1 image per minute (we find that CARS imaging in live cells is slower than in dried, fixed cells).

CARS microscopy is limited to monitoring one vibrational mode at a time, rather than comparing the full spectral signature. However, the RNA and DNA peaks have been shown to drop considerably, and RNA is the dominant change to spectra. So it is possible that CARS imaging will be able to measure the stage of differentiation purely by monitoring the size of each nucleus. White light imaging is less exact at measuring the nucleus size than either fluorescence or CARS microscopy and could not be extended from monolayers; so CARS is preferred as a noninvasive technique to monitor nuclear size. We expect that CARS should also be able to map mineralization from mesenchymal stem cells, by mapping the peak at $960 \mathrm{~cm}^{-1}$. The clear advantage of CARS is its high speed compared to the other spectroscopic techniques.

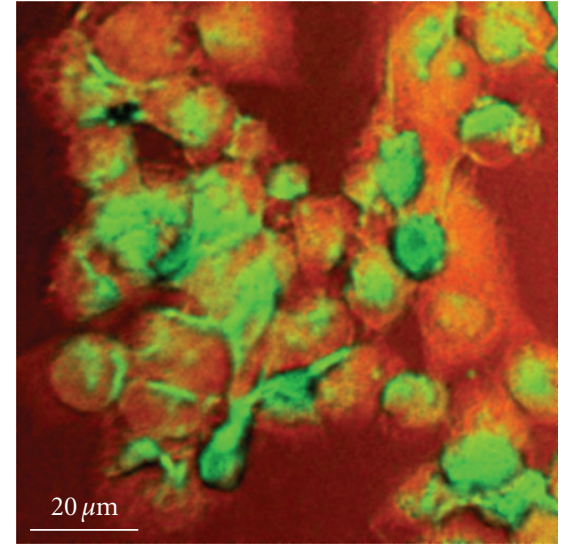

FIGURE 6: CARS microscopy image of fixed MCF-7 breast cancer cells, of size $100 \times 100 \mu \mathrm{m}$. The green channel is specific to the $\mathrm{O}-\mathrm{P}-\mathrm{O}$ stretch frequency $\left(1095 \mathrm{~cm}^{-1}\right)$ and originates from the DNA backbone, so highlights the nucleus (whose shape may have been distorted in some cells, by the fixation process). The red channel is specific to the $\mathrm{CH}_{2}$ deformation $\left(1448 \mathrm{~cm}^{-1}\right)$ and is dominated by lipids and the cytoskeleton. The image plane is restricted to a $1 \mu \mathrm{m}$ slice, acquired several micrometres above the glass substrate, and the lateral resolution is around $350 \mathrm{~nm}$. Images of both channels were acquired in 1 second, and averaged 5 times; both channels were acquired sequentially after retuning one laser source. Pulse widths of 6 ps correspond to a spectral resolution of $\sim 3 \mathrm{~cm}^{-1}$.

One of the most promising techniques over the coming years should be Multiplex CARS, which acquires a full vibrational spectrum at higher speed than Raman, and is also applicable to the single cell level. This is bound to be more sensitive than monitoring just one peak in standard CARS. If the spectral acquisition speed is improved to the millisecond scale, the technology could be applied to both flow cytometry and microscopy.

\section{Conclusions}

The major noninvasive optical spectroscopy techniques suitable for analysis of stem cell differentiation have been outlined: namely, FTIR, Raman, and CARS. FTIR spectroscopy has only been performed on fixed or dried stem cells, but ATR-FTIR can be used in order to investigate live cells in future. Raman spectroscopy has demonstrated the ability to distinguish between live stem cells and differentiated cells: both murine and human embryonic stem cells display a large reduction in peak intensities of both RNA and DNA. When mesenchymal stem cells differentiate into osteoblasts, they display a clear peak (or peaks) relating solely to mineral composition.

It is well known that the nucleus to cytoplasm (volume) ratio in embryonic stem cells is elevated [51-54]. Hence, the reduction in RNA and DNA levels during differentiation is not entirely surprising. The assumption is therefore that the nucleus volume shrinks by around 50\% during differentiation [53] — rather than the concentration of nucleic acids reducing. CARS microscopy is well suited to monitoring the 
nucleus size or mineralization content of each individual cell (around $960 \mathrm{~cm}^{-1}$ ) and can be applied to large numbers of cells in cultures and to engineered tissue scaffolds. Monitoring the CARS signal of the RNA or DNA peak could also be applied to high-speed cell flow cytometry. White light imaging (normally DIC or phase contrast) could offer a lowcost solution for monitoring the nucleus/cytoplasm ratio of cell monolayers, in conjunction with automated image recognition cytometry. CARS only monitors one (or possibly two) spectral peaks; so it will be less sensitive than Raman and FTIR which both acquire a full vibrational spectrum. Multiplex CARS can acquire a full spectrum and promises to replace Raman spectroscopy in time, due to its improved speed.

Each spectroscopic technique has its own benefits and drawbacks; so it is more suited to characterization of stem cells in different ways and on different "platforms." Raman and FTIR spectroscopy are both most suited to monitoring cultures averaged over a large number cells. FTIR does not have the required resolution to address single cells in confluent monolayer cultures, or a sufficient penetration depth for flow cytometry. Raman spectroscopy is too slow to characterize enough individual cells to be a worthwhile clinical technique but could be used extensively in biomedical research. Both FTIR and Raman techniques are more sensitive than normal CARS which relies on excitation of just one spectral peak. However, CARS may be sufficiently sensitive to apply to characterization of differentiation in microscopy and flow cytometry. Raman and CARS have been integrated into one instrument, to combine the benefits of both techniques [55]. In future, Multiplex CARS promises to be the technique of choice for all platforms, due to its combined attributes of speed, full spectral analysis, and applicability to individual live cells.

\section{Acknowledgments}

The authors would like to thank Owen Hughes and Brendon Noble (Centre for Regenerative Medicine, University of Edinburgh) for providing mesenchymal stem cells. A. Downes is supported by a Research Councils UK fellowship, and an award from the Medical Research Council (G0802632). R. Mouras and the CARS microscope are both funded by the EPSRC (EP/E007864/1).

\section{References}

[1] V. F. Segers and R. T. Lee, "Stem-cell therapy for cardiac disease," Nature, vol. 451, no. 7181, pp. 937-942, 2008.

[2] R. Langer, "Tissue engineering: perspectives, challenges, and future directions," Tissue Engineering, vol. 13, no. 1, pp. 1-2, 2007.

[3] L. M. Hoffman and M. K. Carpenter, "Characterization and culture of human embryonic stem cells," Nature Biotechnology, vol. 23, no. 6, pp. 699-708, 2005.

[4] K. Nagano, Y. Yoshida, and T. Isobe, "Cell surface biomarkers of embryonic stem cells," Proteomics, vol. 8, no. 19, pp. 40254035, 2008.
[5] H. Fukuda, J. Takahashi, K. Watanabe, et al., "Fluorescenceactivated cell sorting-based purification of embryonic stem cell-derived neural precursors averts tumor formation after transplantation," Stem Cells, vol. 24, no. 3, pp. 763-771, 2006.

[6] J. Pruszak, K.-C. Sonntag, M. H. Aung, R. Sanchez-Pernaute, and O. Isacson, "Markers and methods for cell sorting of human embryonic stem cell-derived neural cell populations," Stem Cells, vol. 25, no. 9, pp. 2257-2268, 2007.

[7] V. Dousset, T. Tourdias, B. Brochet, C. Boiziau, and K. G. Petry, "How to trace stem cells for MRI evaluation?" Journal of the Neurological Sciences, vol. 265, no. 1-2, pp. 122-126, 2008.

[8] R. C. Addis, J. W. Bulte, and J. D. Gearhart, "Special cells, special considerations: the challenges of bringing embryonic stem cells from the laboratory to the clinic," Clinical Pharmacology and Therapeutics, vol. 83, no. 3, pp. 386-389, 2008.

[9] J. W. Chan, D. K. Lieu, T. Huser, and R. A. Li, "Label-free separation of human embryonic stem cells and their cardiac derivatives using Raman spectroscopy," Analytical Chemistry, vol. 81, no. 4, pp. 1324-1331, 2009.

[10] M. J. Walsh, A. Hammiche, T. G. Fellous, et al., "Tracking the cell hierarchy in the human intestine using biochemical signatures derived by mid-infrared microspectroscopy," Stem Cell Research, vol. 3, no. 1, pp. 15-27, 2009.

[11] M. J. German, H. M. Pollock, B. Zhao, et al., "Characterization of putative stem cell populations in the cornea using synchrotron infrared microspectroscopy," Investigative Ophthalmology \& Visual Science, vol. 47, no. 6, pp. 2417-2421, 2006.

[12] E. V. Loewenstein, "The history and current status of Fourier transform spectroscopy," Applied Optics, vol. 5, no. 5, pp. 845854, 1966.

[13] W. Yang, X. Xiao, J. Tan, and Q. Cai, "In situ evaluation of breast cancer cell growth with 3D ATR-FTIR spectroscopy," Vibrational Spectroscopy, vol. 49, no. 1, pp. 64-67, 2009.

[14] M. J. Tobin, M. A. Chesters, J. M. Chalmers, et al., "Infrared microscopy of epithelial cancer cells in whole tissues and in tissue culture, using synchrotron radiation," Faraday Discussions, vol. 126, pp. 27-39, 2004.

[15] C. Krafft, R. Salzer, S. Seitz, C. Ern, and M. Schieker, "Differentiation of individual human mesenchymal stem cells probed by FTIR microscopic imaging," The Analyst, vol. 132, no. 7, pp. 647-653, 2007.

[16] T. Hirschfeld, "Subsurface layer studies by attenuated total reflection Fourier transform spectroscopy," Applied Spectroscopy, vol. 31, no. 4, pp. 289-292, 1977.

[17] M. K. Kuimova, K. L. A. Chan, and S. G. Kazarian, "Chemical imaging of live cancer cells in the natural aqueous environment," Applied Spectroscopy, vol. 63, no. 2, pp. 164-171, 2009.

[18] C. V. Raman and K. S. Krishnan, "The negative absorption of radiation," Nature, vol. 122, no. 3062, pp. 12-13, 1928.

[19] Y. Liu, G. J. Sonek, M. W. Berns, and B. J. Tromberg, "Physiological monitoring of optically trapped cells: assessing the effects of confinement by 1064-nm laser tweezers using microfluorometry," Biophysical Journal, vol. 71, no. 4, pp. 2158-2167, 1996.

[20] K. Hamada, K. Fujita, N. I. Smith, M. Kobayashi, Y. Inouye, and S. Kawata, "Raman microscopy for dynamic molecular imaging of living cells," Journal of Biomedical Optics, vol. 13, no. 4, Article ID 044027, 4 pages, 2008.

[21] C. Matthäus, T. Chernenko, A. Kale, V. Torchilin, and M. Diem, "New ways of imaging uptake and intracellular fate of liposomal drug carrier systems inside individual cells, based on Raman microscopy," Molecular Pharmaceutics, vol. 5, no. 2, pp. 287-293, 2008. 
[22] C. Krafft, T. Knetschke, A. Siegner, R. H. W. Funk, and R. Salzer, "Mapping of single cells by near infrared Raman microspectroscopy," Vibrational Spectroscopy, vol. 32, no. 1, pp. 75-83, 2003.

[23] V. V. Pully, A. Lenferink, and C. Otto, "Hybrid Rayleigh, Raman and two-photon excited fluorescence spectral confocal microscopy of living cells," to appear in Journal of Raman Spectroscopy.

[24] F. L. Martin, M. J. German, E. Wit, T. Fearn, N. Ragavan, and H. M. Pollock, "Identifying variables responsible for clustering in discriminant analysis of data from infrared microspectroscopy of a biological sample," Journal of Computational Biology, vol. 14, no. 9, pp. 1176-1184, 2007.

[25] P. D. Maker and R. W. Terhune, "Study of optical effects due to an induced polarization third order in the electric field strength," Physical Review, vol. 137, no. 3, pp. A801-A818, 1965.

[26] A. Zumbusch, G. R. Holtom, and X. S. Xie, "Threedimensional vibrational imaging by coherent anti-Stokes Raman scattering," Physical Review Letters, vol. 82, no. 20, pp. 4142-4145, 1999.

[27] C. L. Evans, E. O. Potma, M. Puoris'haag, D. Côté, C. P. Lin, and X. S. Xie, "Chemical imaging of tissue in vivo with videorate coherent anti-Strokes Raman scattering microscopy," Proceedings of the National Academy of Sciences of the United States of America, vol. 102, no. 46, pp. 16807-16812, 2005.

[28] A. Downes, R. Mouras, and A. Elfick, "A versatile CARS microscope for biological imaging," Journal of Raman Spectroscopy, vol. 40, no. 7, pp. 757-762, 2009.

[29] K. König, T. W. Becker, P. Fischer, I. Riemann, and K.J. Halbhuber, "Pulse-length dependence of cellular response to intense near-infrared laser pulses in multiphoton microscopes," Optics Letters, vol. 24, no. 2, pp. 113-115, 1999.

[30] H. Kano and H.-O. Hamaguchi, "In-vivo multi-nonlinear optical imaging of a living cell using a supercontinuum light source generated from a photonic crystal fiber," Optics Express, vol. 14, no. 7, pp. 2798-2804, 2006.

[31] H. Kano and H.-O. Hamaguchi, "Dispersion-compensated supercontinuum generation for ultrabroadband multiplex coherent anti-Stokes Raman scattering spectroscopy," Journal of Raman Spectroscopy, vol. 37, no. 1-3, pp. 411-415, 2006.

[32] H. N. Paulsen, K. M. Hilligse, J. Thøgersen, S. R. Keiding, and J. J. Larsen, "Coherent anti-Stokes Raman scattering microscopy with a photonic crystal fiber based light source," Optics Letters, vol. 28, no. 13, pp. 1123-1125, 2003.

[33] T. W. Kee and M. T. Cicerone, "Simple approach to one-laser, broadband coherent anti-Stokes Raman scattering microscopy," Optics Letters, vol. 29, no. 23, pp. 2701-2703, 2004.

[34] B. Von Vacano, L. Meyer, and M. Motzkus, "Rapid polymer blend imaging with quantitative broadband multiplex CARS microscopy," Journal of Raman Spectroscopy, vol. 38, no. 7, pp. 916-926, 2007.

[35] C. W. Freudiger, W. Min, B. G. Saar, et al., "Label-free biomedical imaging with high sensitivity by stimulated Raman scattering microscopy," Science, vol. 322, no. 5909, pp. 1857$1861,2008$.

[36] E. O. Potma, C. L. Evans, and X. S. Xie, "Heterodyne coherent anti-Stokes Raman scattering (CARS) imaging," Optics Letters, vol. 31, no. 2, pp. 241-243, 2006.

[37] D. L. Marks, J. B. Geddes III, and S. A. Boppart, "Molecular identification by generating coherence between molecular normal modes using stimulated Raman scattering," Optics Letters, vol. 34, no. 12, pp. 1756-1758, 2009.
[38] D. Ami, T. Neri, A. Natalello, et al., "Embryonic stem cell differentiation studied by FT-IR spectroscopy," Biochimica et Biophysica Acta, vol. 1783, no. 1, pp. 98-106, 2008.

[39] M. J. Walsh, T. G. Fellous, A. Hammiche, et al., "Fourier transform infrared microspectroscopy identifies symmetric $\mathrm{PO}_{2}$-modifications as a marker of the putative stem cell region of human intestinal crypts," Stem Cells, vol. 26, no. 1, pp. 108$118,2008$.

[40] I. Notingher, I. Bisson, A. E. Bishop, W. L. Randle, J. M. P. Polak, and L. L. Hench, "In situ spectral monitoring of mRNA translation in embryonic stem cells during differentiation in vitro," Analytical Chemistry, vol. 76, no. 11, pp. 3185-3193, 2004.

[41] R. M. Salasznyk, R. F. Klees, W. A. Williams, A. Boskey, and G. E. Plopper, "Focal adhesion kinase signaling pathways regulate the osteogenic differentiation of human mesenchymal stem cells," Experimental Cell Research, vol. 313, no. 1, pp. 22-37, 2007.

[42] A. J. Bentley, T. Nakamura, A. Hammiche, et al., "Characterization of human corneal stem cells by synchrotron infrared micro-spectroscopy," Molecular Vision, vol. 13, pp. 237-242, 2007.

[43] I. Notingher, I. Bisson, J. M. Polak, and L. L. Hench, "In situ spectroscopic study of nucleic acids in differentiating embryonic stem cells," Vibrational Spectroscopy, vol. 35, no. 12, pp. 199-203, 2004.

[44] J. W. Chan, D. S. Taylor, and D. L. Thompson, "The effect of cell fixation on the discrimination of normal and leukemia cells with laser tweezers Raman spectroscopy," Biopolymers, vol. 91, no. 2, pp. 132-139, 2009.

[45] N. J. Crane, "A nondestructive method for monitoring in vitro stem cell osteogenic differentiation with Raman spectroscopic mapping," Spectroscopy, vol. 24, no. 3, p. 9, 2009.

[46] G. Pelled, K. Tai, D. Sheyn, et al., "Structural and nanoindentation studies of stem cell-based tissue-engineered bone," Journal of Biomechanics, vol. 40, no. 2, pp. 399-411, 2007.

[47] H. Liu, W. Li, S. Shi, S. Habelitz, C. Gao, and P. DenBesten, "MEPE is downregulated as dental pulp stem cells differentiate," Archives of Oral Biology, vol. 50, no. 11, pp. 923-928, 2005.

[48] E. Azrad, D. Zahor, R. Vago, et al., "Probing the effect of an extract of elk velvet antler powder on mesenchymal stem cells using Raman microspectroscopy: enhanced differentiation toward osteogenic fate," Journal of Raman Spectroscopy, vol. 37, no. 4, pp. 480-486, 2006.

[49] E. Gentleman, R. J. Swain, N. D. Evans, et al., "Comparative materials differences revealed in engineered bone as a function of cell-specific differentiation," Nature Materials, vol. 8, no. 9, pp. 763-770, 2009.

[50] S. O. Konorov, C. H. Glover, J. M. Piret, et al., "In situ analysis of living embryonic stem cells by coherent anti-Stokes Raman microscopy," Analytical Chemistry, vol. 79, no. 18, pp. 72217225, 2007.

[51] J. A. Thomson, J. Itskovitz-Eldor, S. S. Shapiro, et al., "Embryonic stem cell lines derived from human blastocysts," Science, vol. 282, no. 5391, pp. 1145-1147, 1998.

[52] S. K. Oh, S. H. Kim, H. J. Ahn, et al., "Derivation and characterization of new human embryonic stem cell lines: SNUhES1, SNUhES2, and SNUhES3," Stem Cells, vol. 23, no. 2, pp. 211-219, 2005.

[53] I. Faro-Trindade and P. R. Cook, "A conserved organization of transcription during embryonic stem cell differentiation and in cells with high C value," Molecular Biology of the Cell, vol. 17, no. 7, pp. 2910-2920, 2006. 
[54] A. Prowse, E. Wolvetang, and P. Gray, "A rapid, cost-effective method for counting human embryonic stem cell numbers as clumps," BioTechniques, vol. 47, no. 1, pp. 599-606, 2009.

[55] M. N. Slipchenko, T. T. Le, H. Chen, and J.-X. Cheng, "High-speed vibrational imaging and spectral analysis of lipid bodies by compound Raman microscopy," Journal of Physical Chemistry B, vol. 113, no. 21, pp. 7681-7686, 2009. 

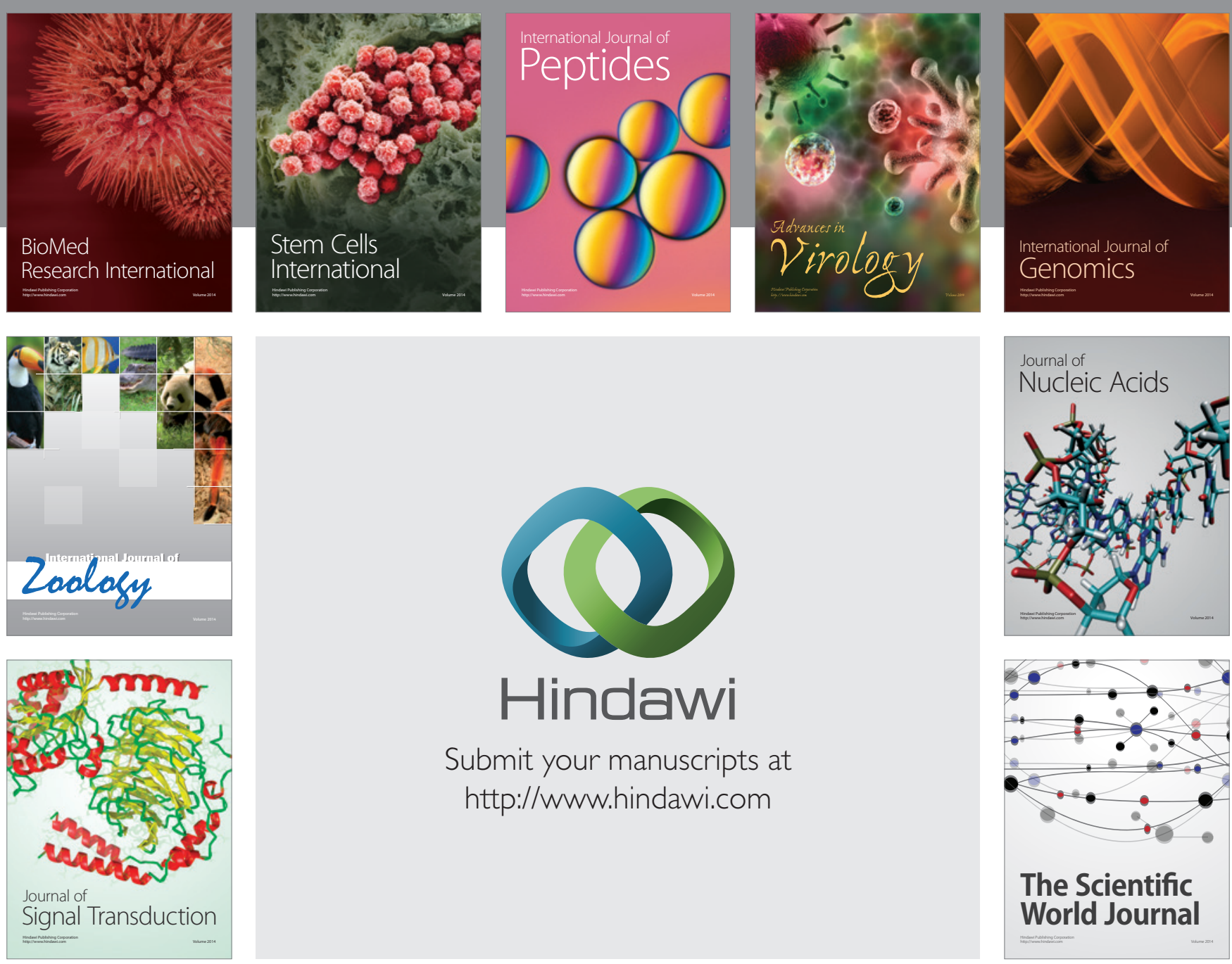

Submit your manuscripts at

http://www.hindawi.com
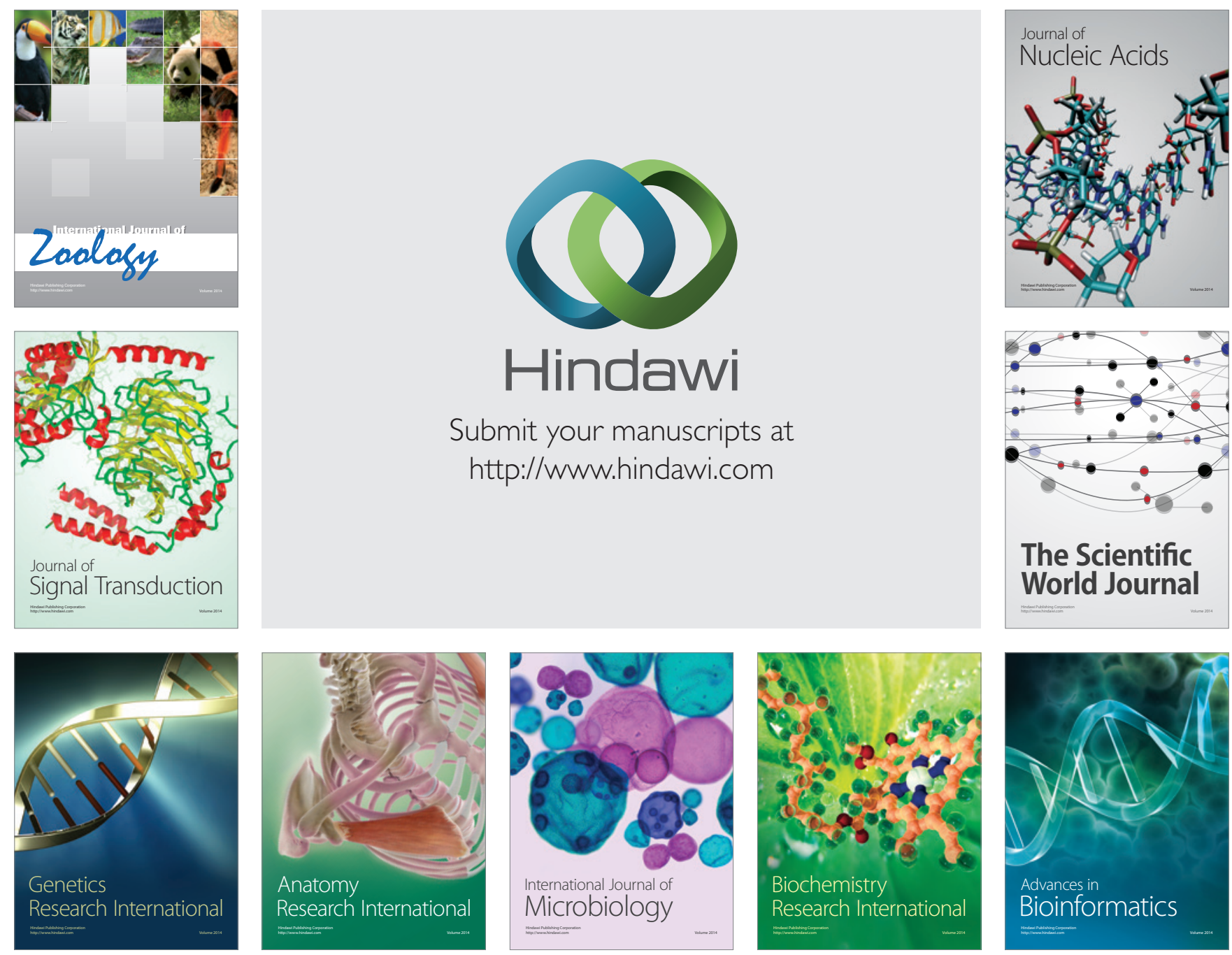

The Scientific World Journal
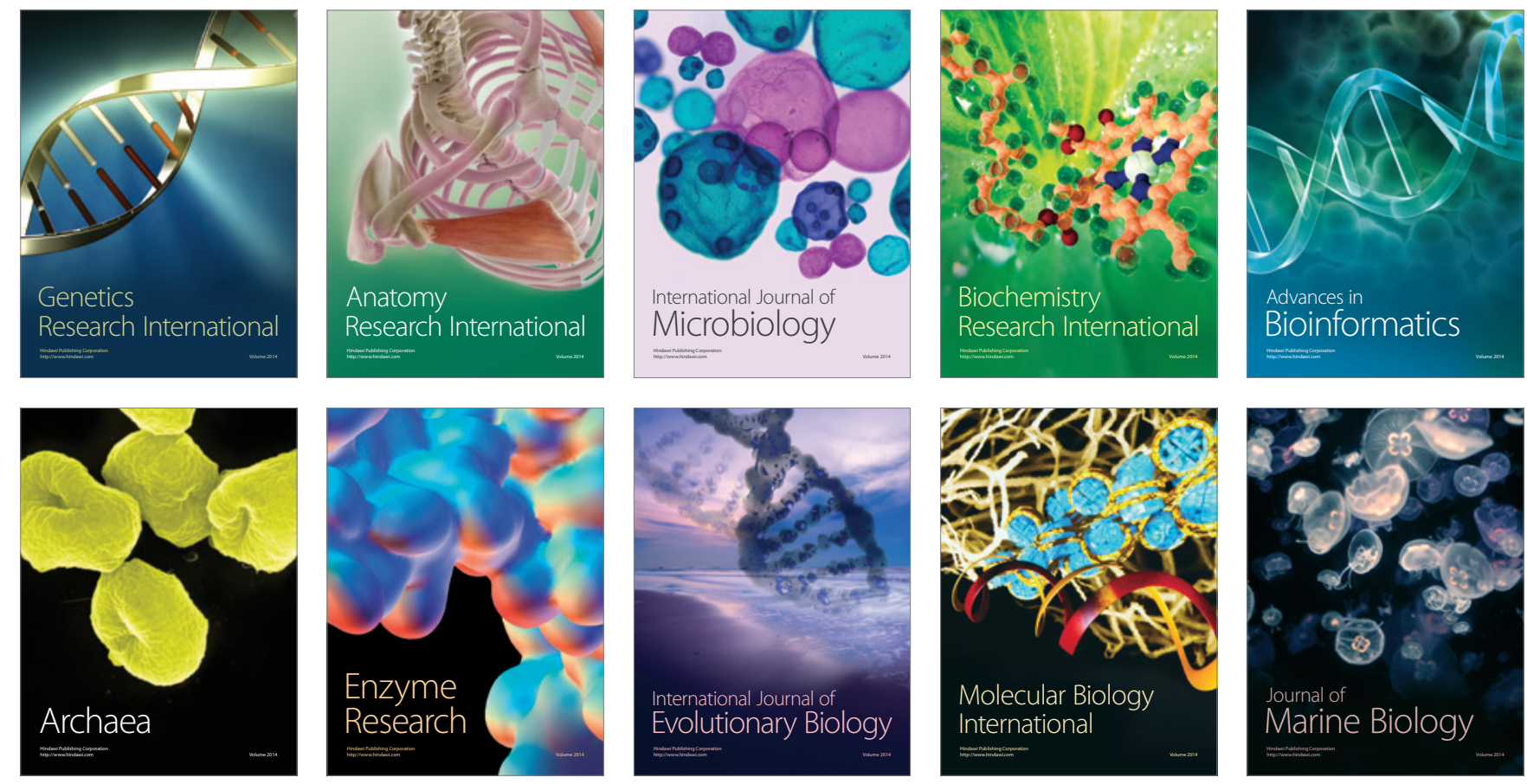